\title{
Optically Active Polymers Containing Side Chain Azobenzene Chromophores: Chiroptical Properties and Photochromic Behaviour of 4-Methacryloxyazobenzene/(-)- Menthyl Methacrylate Copolymers
}

\author{
Angelina Altomare, Carlo Carlini,* Francesco Ciardelli, \\ and Roberto SOLARO \\ Dipartimento di Chimica e Chimica Industriale, \\ Centro CNR Macromolecole Stereordinate Otticamente Attive, \\ University of Pisa, via Risorgimento 35, 56100 Pisa, Italy \\ * Dipartimento di Chimica Industriale e dei Materiali, \\ University of Bologna, \\ viale Risorgimento 4, 40136 Bologna, Italy
}

(Received June 8, 1988)

\begin{abstract}
Copolymers of trans-4-methacryloxyazobenzene (MAB) with (-)-menthyl methacrylate (MtMA) have been prepared by using AIBN as free radical initiator. The resulting polymer samples are characterized by a substantially random distribution of monomeric co-units, as evaluated from reactivity ratios. Kinetics of photoinduced trans to cis and thermally induced cis to trans back-isomerization of azobenzene side chains have been examined and the relevant kinetic parameters evaluated. Dependence of chiroptical properties on both copolymer composition and extent of cis/trans isomerization has been investigated. The circular dichroism induced on azobenzene chromophores has been shown to arise mainly from isolated MAB units, as the chiral perturbation is not appreciably transmitted along MAB sequences. The results have been discussed in terms of tertiary structure reversible variations connected to photoinduced change of local conformation.
\end{abstract}

KEY WORDS (-)-Menthyl Methacrylate / 4-Methacryloxyazobenzene / Photochromic Polymers / cis-trans Isomerization / Chiral Polymers / Chiroptical Properties / Circular Dichroism /

In previous papers ${ }^{1-3}$ it has been reported that copolymers of optically active $(-)$-menthyl acrylate (MtA) with photochromic monomers, such as 4-acryloxyazobenzene (AAB), 4-acryloxystilbene (AS), and 4-vinylstilbene (VS), show dichroic bands in the absorption region of the aromatic groups.

The ellipticity of these bands were found to be dependent on the content of photochromic groups and trans to cis photoisomerization extent. ${ }^{1,4-6}$ In the case of stilbene containing polymers the variation of dichroic properties induced by light irradiation suggested some local conformational change, whereas the re- sults for $\mathrm{AAB} / \mathrm{MtA}$ copolymers appeared less evident. As these last copolymers, prepared by radical initiation, are mainly non stereoregular with a substantial tendency to alternating structure $\left(r_{\mathrm{AAB}} \cdot r_{\mathrm{MtA}}=0.45\right)$, it appeared interesting to investigate the influence of main chain stereoregularity and monomer sequence distribution on photophysical and chiroptical properties.

For both pourposes 4-methacryloxyazobenzene/(-)-menthyl methacrylate copolymers appeared the most convenient. In fact it is well known that in the copolymerization with aromatic monomers, methacrylic esters ex- 
hibit fairly higher reactivity ratios than the corresponding acrylic derivatives. ${ }^{7-10}$ In addition, depending on polymerization conditions, methacrylic polymers can be rather easily prepared with stereoregularity degree ranging from mainly syndiotactic to prevailing isotactic. ${ }^{11,12}$ In this paper the preparation is described of copolymers of 4-methacryloxyazobenzene with (-)-menthyl methacrylate copolymers by free radical polymerization, as well as their chiroptical and photochromic behaviour. The stereoregular copolymers of the same monomers and their properties will be reported in a future paper.

\section{EXPERIMENTAL}

\section{Monomers}

trans-4-Methacryloxyazobenzene (MAB) was synthesized by reacting commercial (Fluka) trans-4-hydroxyazobenzene with methacryloyl chloride and triethylamine (molar ratio $1: 1.5: 2$ ) in anhydrous diethyl ether at room temperature for $6 \mathrm{~h}$. The reaction mixture was extracted with chloroform and the extracts were washed with dilute $\mathrm{HCl}, 5 \%$ $\mathrm{NaHCO}_{3}$, and water, in that order, and dried over anhydrous $\mathrm{Na}_{2} \mathrm{SO}_{4}$. After removal of the solvents the crude product was recrystallized from ethanol/hexane $1: 1$ to give pure MAB (96\% yield) as bright yellow lamellae having mp $116^{\circ} \mathrm{C} .{ }^{1} \mathrm{H}$ NMR $\left(\mathrm{CDCl}_{3}\right): \delta=7.95,7.50$ and $7.28(3 \mathrm{~m}, 9 \mathrm{H}$, aromatic protons), 6.38 and $5.78(2 \mathrm{~m}, 2 \mathrm{H}$, olefinic protons) and $2.07 \mathrm{ppm}$ (m, 3H, methyl protons). IR (KBr pellet): $\bar{v}=$ $3100-3000\left(v_{\mathrm{CH}}\right.$ aromatic), 3000-2900 $\left(v_{\mathrm{CH}}\right.$ aliphatic), $1782\left(v_{\mathrm{C}=\mathrm{O}}\right), 1637\left(v_{\mathrm{C}=\mathrm{C}}\right.$ olefinic $)$, 1580,1490 , and $1480\left(v_{\mathrm{C}=\mathrm{C}}\right.$ aromatic $), 1380$ $\left(\delta_{\mathrm{s}} \mathrm{CH}_{3}\right), 1195\left(v_{\mathrm{as}} \mathrm{C}-\mathrm{O}-\mathrm{C}\right), 1120\left(v_{\mathrm{s}} \mathrm{C}-\mathrm{O}-\mathrm{C}\right)$, $890\left(\delta_{\mathrm{CH}_{2}}=\right), 815\left(\delta_{\mathrm{CH}} 1,4\right.$-disubstituted phenyl), 765 and $685 \mathrm{~cm}^{-1}\left(\delta_{\mathrm{CH}}\right.$ monosubstituted phenyl).

(-)-Methyl methacrylate (MtMA) having bp $95^{\circ} \mathrm{C} / 2 \mathrm{~mm}$ and $[\alpha]_{\mathrm{D}}^{25}=-91.8$ (neat), was prepared in $89 \%$ yield starting from (-)menthol and following the above procedure.
trans-4-(2-Methylpropanoyl)azobenzene (IAB) was synthesized as previously reported. ${ }^{1}$ Commercial 2,2'-Azobisisobutyronitrile (AIBN) was recrystallized from ethanol.

\section{Polymerization Experiments}

Free radical copolymerization experiments were performed in benzene at $60^{\circ} \mathrm{C}$ in vials sealed under high vacuum using AIBN as initiator. After a suitable period of time, polymerization was interrupted by pouring the reaction mixture into a large excess of methanol. The coagulated polymer was purified by repeated precipitation in methanol. Finally, the polymeric product was filtered and dried under vacuum at room temperature.

\section{Physicochemical Measurements}

200-MHz ${ }^{1} \mathrm{H}$ NMR spectra were recorded in $\mathrm{CDCl}_{3}$ solution with a Varian Gemini-200 spectrometer using TMS as the internal standard. IR examinations were carried out with a Perkin-Elmer 1330 spectrophotometer. UV and $\mathrm{CD}$ measurements in the range 500$250 \mathrm{~nm}$ were performed in $\mathrm{CHCl}_{3}$ solution by a JASCO Uvidec 510 spectrophotometer and a JASCO J500A dichrograph. Optical rotatory measurements were performed on polymer solution in $\mathrm{CHCl}_{3}$ at $25^{\circ} \mathrm{C}$ with a Perkin-Elmer 141 spectropolarimeter. Melting point determinations were carried out on a hot-plate Kofler microscope with a $3^{\circ} \mathrm{C} \mathrm{min}{ }^{-1}$ heating rate. Average molecular weights of polymer samples were determined in THF solution by GPC measurements on a Perkin-Elmer Model 2/2 HPLC, equipped with two Shodex columns A 802/S and 803/S connected in series. Poly(styrene) standards were used for calibration. Irradiations of samples in chloroform solution were carried out at room temperature with a halogen lamp $(150 \mathrm{~W})$ filtered with a $348 \mathrm{~nm}$ interference filter. Thermal cis to trans isomerization experiments were carried out in chloroform distilled over calcium hydride just prior the use and followed by UV at constant temperature. 
Table I. Copolymerization of 4-methacryloxyazobenzene (MAB) with (-)-methyl methacrylate (MtMA) in benzene at $60^{\circ} \mathrm{C}$ with AIBN as initiator ${ }^{\mathrm{a}}$

\begin{tabular}{|c|c|c|c|c|c|c|c|}
\hline \multirow{2}{*}{ Run } & \multirow{2}{*}{$\begin{array}{c}\begin{array}{c}\text { Feed } \\
\text { MAB }\end{array} \\
\mathrm{mol}_{\%}\end{array}$} & \multirow{2}{*}{$\frac{\text { Time }}{\mathrm{h}}$} & \multirow{2}{*}{$\frac{\text { Conv. }^{b}}{\%}$} & \multirow{2}{*}{$\frac{\mathrm{MAB}^{\mathrm{c}}}{\mathrm{mol} \%}$} & \multicolumn{3}{|c|}{ Polymer } \\
\hline & & & & & {$[\alpha]_{D}^{25 d}$} & $\bar{M}_{n}^{\mathrm{e}}$ & $\bar{M}_{w} / \bar{M}_{n}$ \\
\hline MA1 & 100 & 40 & 61.3 & 100 & - & 51000 & 2.1 \\
\hline MA2 & 85 & 5 & 21.1 & 88.6 & -10.1 & 76000 & 2.0 \\
\hline MA3 & 70 & 5 & 20.8 & 74.9 & -25.0 & 88000 & 1.9 \\
\hline MA4 & 50 & 6 & 12.7 & 55.4 & -42.2 & 98000 & 1.7 \\
\hline MA5 & 30 & 5 & 14.2 & 38.1 & -61.5 & 96000 & 1.7 \\
\hline MA6 & 15 & 2 & 3.9 & 19.0 & -83.5 & 104000 & 1.7 \\
\hline MA7 & 5 & 4 & 22.1 & 7.1 & -89.0 & 113000 & 1.6 \\
\hline MA8 & 0 & 40 & 80.0 & 0 & -94.2 & 108000 & 1.7 \\
\hline
\end{tabular}

a Benzene $25 \mathrm{ml}$, comonomer mixture $3 \mathrm{~g}$, AIBN $0.03 \mathrm{~g}$.

b Evaluated as (weight of polymer/total weight of starting comonomers) $\times 100$.

c Evaluated by ${ }^{1} \mathrm{H}$ NMR.

d In chloroform.

e Determined by GPC.

\section{RESULTS AND DISCUSSION}

\section{Synthesis and Characterization of Copolymers}

Optically active copolymers of (-)-menthyl methacrylate with 4-methacryloxyazobenzene [poly(MtMA-co-MAB)s] were prepared by polymerization in benzene at $60^{\circ} \mathrm{C}$ in the presence of AIBN as radical initiator; starting molar ratios $\mathrm{MAB} / \mathrm{MtMA}$ in the range $6-$ 0.05 were used (Table I). Copolymerization runs were interrupted at low conversion in order to determine the comonomer reactivity ratios and to dispose of copolymer samples having substantially homogeneous composition and distribution of monomeric units. The copolymerization diagram (Figure 1) indicates that MAB is slightly more reactive than MtMA. The reactivity ratios calculated by least-square evaluation of the Kelen-Tudos parameters $^{13}$ are $r_{\mathrm{MAB}}=1.27$ and $r_{\mathrm{MtMA}}=0.7$. The product of these reactivity ratios is quite close to unit $\left(r_{\mathrm{MtMA}} \cdot r_{\mathrm{MAB}}=0.89\right)$ and therefore the copolymer samples are characterized by a substantially random distribution of monomeric units. ${ }^{14}$ However a better insight into the chemical structure of different copolymer

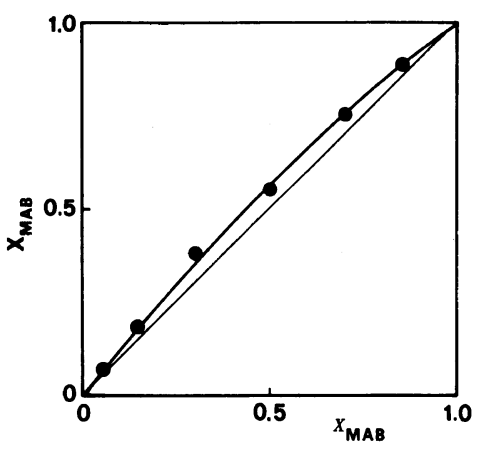

Figure 1. Copolymerization diagram for the MtMA/ MAB system: $X_{\text {MAB }}$ and $X_{\text {MAB }}$ are the MAB molar fractions in the feed mixture and in the copolymerization products, respectively.

samples can be obtained from the average sequence lengths $(\bar{l})$ and percent molar fractions of units $[X(n)]$ inserted in blocks on $n$ units (Table II) as evaluated from the copolymerization data. ${ }^{15,16}$

Fairly high molecular weights $\left(5-11 \times 10^{4}\right)$ are observed in all cases. The dependence of both molecular weight $\left(\bar{M}_{n}\right)$ and degree of dispersion $\left(\bar{M}_{w} / \bar{M}_{n}\right)$ on the composition of the copolymerization mixture seems to indicate a higher tendency of MAB, as compared with 


\section{A. Altomare et al.}

Table II. Mean sequence lengths $\left(\bar{l}_{\mathrm{MAB}}\right.$ and $\left.\bar{l}_{\mathrm{MtMA}}\right)$ and percent molar fractions $\left[X_{\mathrm{MAB}}(n)\right.$ and $\left.X_{\mathrm{MtMA}}(n)\right]$ of units from MAB and MtMA in sequence lengths of $n$ units for poly(MtMA-co-MAB)s ${ }^{\mathrm{a}}$

\begin{tabular}{|c|c|c|c|c|c|c|c|c|c|c|c|c|c|c|c|}
\hline \multirow{2}{*}{ Sample } & \multirow{2}{*}{$\frac{\text { MAB }}{\mathrm{mol} \%}$} & \multirow{2}{*}{$\bar{l}_{\mathrm{MAB}}$} & \multirow{2}{*}{$\bar{l}_{\text {MtMA }}$} & \multicolumn{6}{|c|}{$X_{\mathrm{MAB}}(n)$} & \multicolumn{6}{|c|}{$X_{\mathrm{MtMA}}(n)$} \\
\hline & & & & $n=1$ & 2 & 3 & 4 & 5 & $>5$ & $n=1$ & 2 & 3 & 4 & 5 & $>5$ \\
\hline MA2 & 88.6 & 10.9 & 1.1 & 0.8 & 1.5 & 2.1 & 2.5 & 2.9 & 90.2 & 84.2 & 13.9 & 1.7 & 0.2 & 0.0 & 0.0 \\
\hline MA3 & 74.9 & 4.8 & 1.2 & 4.4 & 6.9 & 8.2 & 8.6 & 8.5 & 63.4 & 65.6 & 24.9 & 7.1 & 1.8 & 0.4 & 0.2 \\
\hline MA4 & 55.4 & 2.6 & 1.6 & 15.1 & 18.4 & 16.9 & 13.8 & 10.6 & 25.2 & 40.9 & 29.5 & 15.9 & 7.7 & 3.5 & 2.5 \\
\hline MA5 & 38.1 & 1.8 & 2.1 & 31.5 & 27.6 & 18.2 & 10.6 & 5.8 & 6.3 & 21.9 & 23.3 & 18.6 & 13.2 & 8.8 & 14.2 \\
\hline MA6 & 19.0 & 1.3 & 4.0 & 59.4 & 27.3 & 9.4 & 2.9 & 0.8 & 0.2 & 6.3 & 9.4 & 10.6 & 10.6 & 9.9 & 52.2 \\
\hline MA7 & 7.1 & 1.1 & 10.2 & 83.1 & 14.7 & 2.0 & 0.2 & 0.0 & 0.0 & 1.0 & 1.7 & 2.4 & 2.8 & 3.2 & 88.9 \\
\hline
\end{tabular}

a Evaluated according to ref 15 and 16.

MtMA, to promote chain transfer and/or termination during the polymerization process.

IR spectra of poly(MtMA-co-MAB)s show the same bands as the corresponding homopolymers mixtures, indicating that both counits are present in the macromolecules. On the other hand solubility and chiroptical properties (see below) rule out the formation of homopolymer mixtures.

In the region of aromatic protons the ${ }^{1} \mathrm{H}$ NMR spectrum of IAB is characterized by three sets of signals centered at 7.22, 7.46, and $7.93 \mathrm{ppm}$. Correspondingly the spectra of copolymer samples display three broad bands centered at $7.21,7.36$, and $7.80 \mathrm{ppm}$. Two small bands are also observed at about 6.95 and $6.75 \mathrm{ppm}$, whose relative intensity decreases with decreasing the MAB content. As a consequence these two higher field signals have been attributed to partially overlapping MAB chromophores, in close analogy to what already reported for several copolymers of vinylaromatic monomers. ${ }^{17}$ However the small relative intensity of these bands, even in the MAB homopolymer (about $0.4 \mathrm{H}$ ), suggests that the extent of interaction between side chain azobenzene groups should be rather limited, at least in the NMR time scale.

\section{Reversible trans-cis Isomerization}

By irradiation at $348 \mathrm{~nm}$ the investigated polymer samples and the low molecular weight

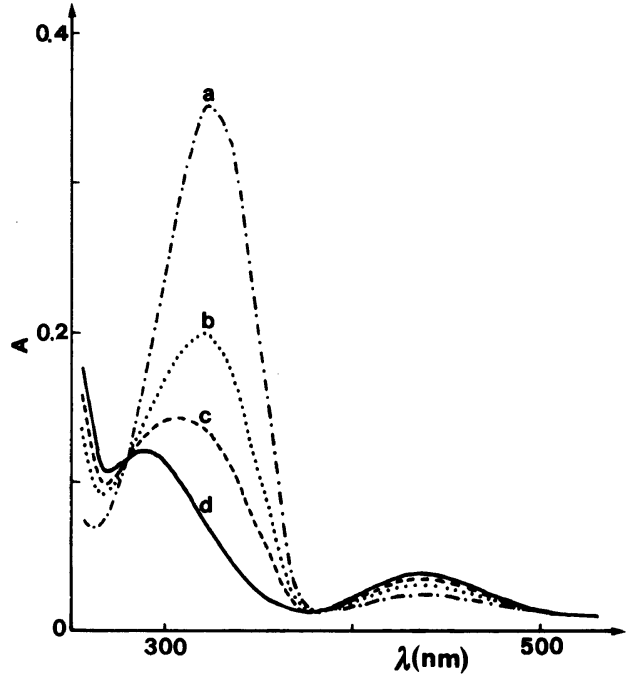

Figure 2. Variation of the UV absorption spectrum in chloroform solution of a MtMA/MAB copolymer sample containing $7.1 \% \mathrm{~mol}$ of MAB units with irradiation time at $348 \mathrm{~nm}$. Curves a to $\mathrm{d}$ correspond to 0 , 30,60 , and $300 \mathrm{~s}$ of irradiation time, respectively.

structural analog IAB undergo trans to cis photoisomerization until a photostationary state is eventually reached (Figure 2). In all cases a plot of $A_{\mathrm{r}}$, where $A_{\mathrm{r}}$ is equal to $\ln \left[\left(A_{0}-A_{\infty}\right) /\left(A_{\mathrm{t}}-A_{\infty}\right)\right]$, and $A_{0}, A_{\mathrm{t}}$, and $A_{\infty}$ are the absorbances at $320 \mathrm{~nm}$ at time $0, t$ and $\infty$, respectively, $v s$. the irradiation time fits a first-order kinetics. ${ }^{4}$ Photoisomerization rates and the ratio $A_{\infty} / A_{0}$ of polymer samples are of the same order of magnitude as those observed 
Table III. UV absorption properties and photochromism of high and low molecular weight trans-azobenzene derivatives

\begin{tabular}{|c|c|c|c|c|c|}
\hline \multirow{3}{*}{ Sample } & \multirow{3}{*}{$\frac{\mathrm{MAB}}{\mathrm{mol} \%}$} & \multicolumn{2}{|c|}{$U^{a}$} & \multirow{3}{*}{$\begin{array}{l}\text { Photoisomerization }{ }^{b} \\
\frac{\text { rate constant } \times 10^{2}}{\mathrm{~s}^{-1}}\end{array}$} & \multirow{3}{*}{$A_{\infty} / A_{0}$} \\
\hline & & \multirow{2}{*}{$\frac{\lambda_{\max }}{\mathrm{nm}}$} & \multirow{2}{*}{$\frac{\varepsilon_{\max }}{\mathrm{mol} \cdot 1^{-1} \cdot \mathrm{cm}^{-1}}$} & & \\
\hline & & & & & \\
\hline MAl & 100 & 321.5 & 18800 & 2.1 & 0.25 \\
\hline MA2 & 88.6 & 322.0 & 18900 & 2.2 & 0.26 \\
\hline MA3 & 74.9 & 322.0 & 18000 & 2.2 & 0.25 \\
\hline MA4 & 55.4 & 322.5 & 21000 & 2.2 & 0.25 \\
\hline MA5 & 38.1 & 322.6 & 21500 & 2.4 & 0.26 \\
\hline MA6 & 19.0 & 323.5 & 23000 & 2.4 & 0.25 \\
\hline MA7 & 7.1 & 324.0 & 23800 & 2.7 & 0.23 \\
\hline IAB & - & 324.0 & 22800 & 2.3 & 0.25 \\
\hline
\end{tabular}

a In chloroform solution at room temperature.

b Under irradiation at $348 \mathrm{~nm}$ in chloroform solution at room temperature.

for IAB (Table III). This indicates that the macromolecular structure has little influence on the photoisomerization process, analogously to what observed for several azobenzene containing polymers. ${ }^{4,5,18}$

When kept in the dark copolymer samples mainly containing cis-azobenzene moieties undergo thermal cis to trans back-isomerization. The isomerization rates heavily depend on the purity of the chloroform used as solvent. In fact the addition of a small amount of $\mathrm{HCl}$ gives rise to an increase of two order of magnitude whereas the presence of $0.75 \%$ of ethanol as stabilizer gives rise to both an increase of the rate and a departure from first order kinetics (Figure 3).

The linear dependence of $A_{\mathrm{r}}$ on time is also in this case in accordance with a first order kinetics. The evaluated isomerization rates are almost independent of the copolymer composition and close to that of IAB (Table IV). An Arrhenius plot of the reaction rates in the range $25-55^{\circ} \mathrm{C}$ gives straight lines (Figure 4) and allows one to evaluate a pre-exponential factor $C$ of about $2.5 \times 10^{7} \mathrm{~s}^{-1}$ and an activation energy $\Delta E^{*}$ of about $17 \mathrm{kcal} \mathrm{mol}^{-1}$ for the investigated samples (Table IV).

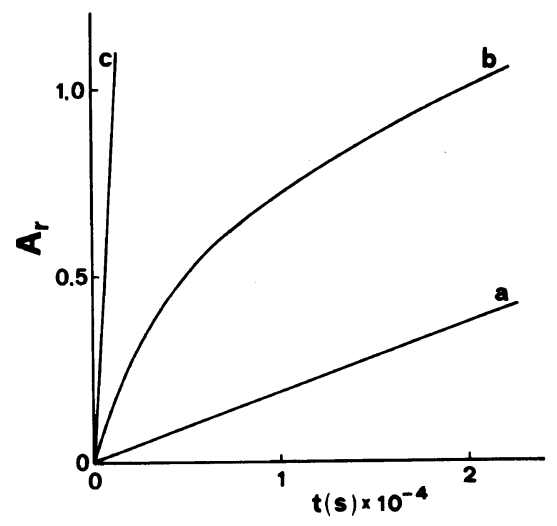

Figure 3. Kinetic plot of thermal cis-trans isomerization in chloroform solution at $35^{\circ} \mathrm{C}$ of poly(MtMA-coMAB) samples containing $7.1 \% \mathrm{~mol}$ of MAB units: a) solvent purified over $\mathrm{CaH}_{2}$; b) solvent containing $0.75 \%$ of ethanol; c) solvent containing $1 \%$ of concentrated $\mathrm{HCl}$.

\section{Chiroptical Properties}

The UV spectrum of all the investigated samples is characterized, in the $550-250 \mathrm{~nm}$ region, by two absorption bands centered at about 440 and $322 \mathrm{~nm}$ (Figure 2) and attributed to $n \rightarrow \pi^{*}$ and $\pi \rightarrow \pi^{*}$ electronic transitions of azobenzene chromophore, respectively. ${ }^{19}$

As compared with the low molecular weight 
A. Altomare et al.

Table IV. Thermal isomerization of polymers containing MAB structural units and their low molecular weight model compound IAB

\begin{tabular}{|c|c|c|c|c|c|c|c|}
\hline \multirow{2}{*}{ Sample } & \multirow{2}{*}{$\frac{\mathrm{MAB}}{\mathrm{mol} \%}$} & \multicolumn{4}{|c|}{$\begin{array}{l}\text { Isomerization rate } \\
\text { constant } \times 10^{5}\left(\mathrm{~s}^{-1}\right)\end{array}$} & \multirow{2}{*}{$\frac{\Delta E^{* \mathrm{a}}}{\mathrm{kcal} \mathrm{mol}^{-1}}$} & \multirow{2}{*}{$\ln C^{\mathrm{a}}$} \\
\hline & & $25^{\circ} \mathrm{C}$ & $35^{\circ} \mathrm{C}$ & $45^{\circ} \mathrm{C}$ & $55^{\circ} \mathrm{C}$ & & \\
\hline MA1 & 100 & 0.83 & 2.3 & 4.3 & 12 & 16.7 & 17 \\
\hline MA2 & 88.6 & 0.85 & 2.1 & 4.3 & 12 & 16.7 & 17 \\
\hline MA3 & 74.9 & 0.90 & 2.1 & 4.3 & 13 & 16.8 & 17 \\
\hline MA4 & 55.4 & 0.86 & 2.0 & 4.3 & 13 & 17.2 & 17 \\
\hline MA5 & 38.1 & 0.84 & 2.0 & 4.3 & 13 & 17.3 & 18 \\
\hline MA6 & 19.0 & 0.80 & 2.0 & 4.3 & 13 & 17.6 & 18 \\
\hline MA7 & 7.1 & 0.77 & 1.9 & 4.4 & 13 & 18.1 & 19 \\
\hline IAB & - & 0.76 & 2.2 & 3.6 & 9 & 15.5 & 15 \\
\hline
\end{tabular}

a Evaluated from the relation $\ln k=\ln C-\Delta E^{*} / R T$.

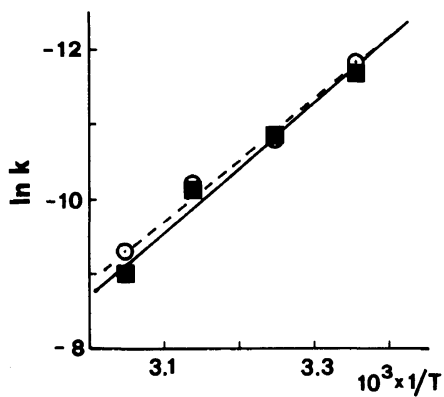

Figure 4. Arrhenius plot of cis to trans thermal isomerization rates of (- - ) poly(MtMA-co-MAB)s and $(-\odot-)$ IAB.

model compound IAB, polymer samples exhibit progressive hypocromic and hypsochromic effects with increasing content of MAB units (Table III). A similar trend has already been observed in several polymers containing aromatic chromophores ${ }^{17,20}$ and has been attributed to electronic interactions between neighbouring chromophores. ${ }^{21,22}$

All copolymer samples are characterized by a negative optical rotation at sodium $D$ line, as observed for poly(MtMA) (Table I). A plot of specific rotation $[\alpha]_{\mathrm{D}}^{25} v s$. the content of MtMA units exhibits a linear trend thus excluding any contribution of the MAB units to optical rotation at $589 \mathrm{~nm}$. A similar behavior has already been observed in other optically active copolymers of (-)-menthyl acrylate and methacrylate with unsaturated aromatic monomers. $^{2,7,23.24}$ This finding does not exclude an asymmetric perturbation of the azobenzene moiety and indeed CD spectra show in the $550-250 \mathrm{~nm}$ region one broad, negative dichroic band centered at about $450 \mathrm{~nm}$ (Figure 5). No dichroic absorption is detectable in the correspondence of the $\pi \rightarrow \pi^{*}$ electronic transition. The absolute value of the ellipticity monotonously increases with the increasing content of MtMA units (Table V) even if no linear dependence on both chemical composition and mean sequence lengths of monomeric units can be detected. On the contrary a plot of $\Delta \varepsilon v s$. the molar fraction of isolated MAB units (Figure 6) fits a straight line, thus suggesting that the observed $C D$ mainly arises from isolated $\mathrm{MAB}$ units, i.e., the induced chiral perturbation is not appreciably transmitted along MAB sequences. A similar behaviour has been already reported for copolymers of (-)-menthyl acrylate with 4hydroxybenzophenone acrylate and has been attributed to the relative conformational mobility of the side chain aromatic chromophore. $^{20}$ 


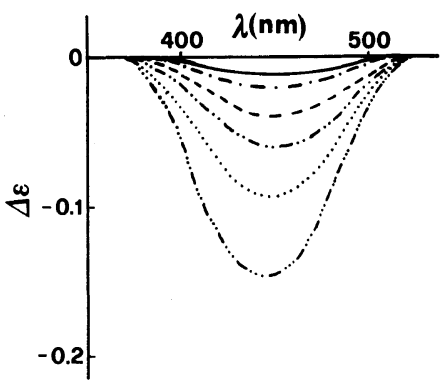

Figure 5. $\mathrm{CD}$ spectra of all trans poly(MtMA-coMAB)s containing, respectively, (---) $7.1 \%$, (-----) $19.0 \%,(---) 38.1 \%,(---) 55.4 \%,(---) 74.9 \%$, and $(-) 88.6 \%$ of MAB units.

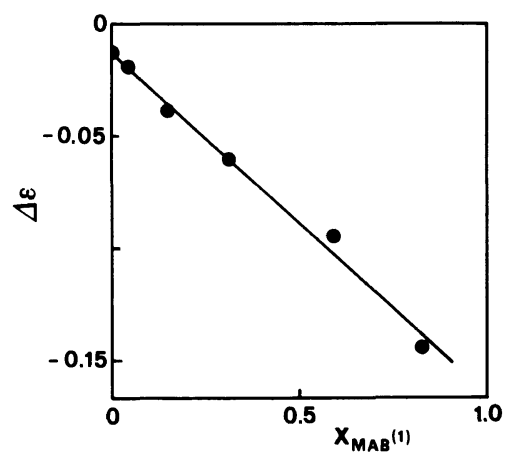

Figure 6. Differential molar dichroic absorption coefficient at $450 \mathrm{~nm}\left(D_{\mathrm{e}}\right)$ of all trans poly(MtMA-co-MAB)s in chloroform solution $v s$. molar fraction of isolated MAB units $\left[X_{\text {MAB }}(1)\right]$.

The CD spectra of analogous copolymers of (-)-menthyl acrylate with 4-acryloxyazobenzene display two dichroic bands centered at about 300 and $435 \mathrm{~nm}^{1}$ The former band has been shown to arise mainly from transazobenzene containing units inserted in blocks and to a minor extent from isolated ones. As in poly(MtMA-co-MAB)s blocks of MAB units are not appreciably chirally perturbed, the contribution to the dichroic absorption at about $300 \mathrm{~nm}$ by interacting trans-azobenzene chromophores should be absent. As a consequence the anisotropy factor $(\Delta \varepsilon / \varepsilon)$ too small to give rise to measurable $C D$.

Spectra of copolymer samples containing MAB units mainly in the cis form exhibit a broad positive dichroic band and a less intense

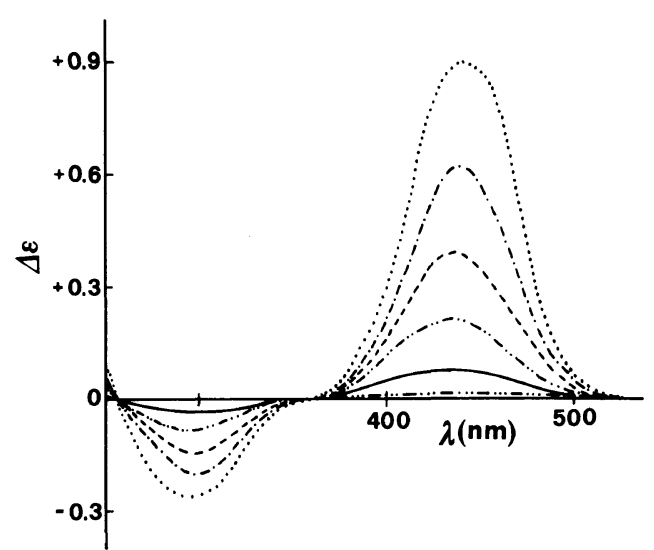

Figure 7. $C D$ spectra at the photostationary state of poly(MtMA-co-MAB)s containing, respectively, (-----) $7.1 \%$, (-- ) $19.0 \%$, (----) $38.1 \%$, (-- -) $55.4 \%$, (-) $74.9 \%$, and (--.) $88.6 \%$ of MAB units.

negative one in the correspondence of $n \rightarrow \pi^{*}$ and $\pi \rightarrow \pi^{*}$ electronic transitions, respectively (Figure 7). Their relative intensity monotonically increases with the increasing content of chiral units (Table V). The positive $\mathrm{CD}$ band at $430 \mathrm{~nm}$ must be attributed to cis MAB units, whereas the dichroic band at $300 \mathrm{~nm}$ can be tentatively assigned to residual trans-azobenzene chromophores, in close analogy to what reported for the corresponding acrylic copolymers. ${ }^{1}$ The appearance of this new band may arise from an increase of the anisotropy factor due to the much lower UV absorption at $300 \mathrm{~nm}$ of $c i s$-azobenzene moieties (Figure 2).

In the case of the sample containing $7.1 \%$ of MAB units, CD spectra have been taken at different cis/trans ratios (Figure 8). The $430 \mathrm{~nm}$ dichroic band turns from negative to positive and then steadily increases with increasing the cis content (Table VI). Correspondingly the negative CD band at $300 \mathrm{~nm}$ becomes detectable, its absolute value increasing with the cis/ trans ratio. The low content of MAB units and the relatively low degree of main chain stereoregularity should rule out the presence of long range cooperative effects. However, the rather sharp variation of the dichroic absorption observed for a relatively small extent of 


\section{A. Altomare et al.}

Table V. Circular dichroism between 500 and $250 \mathrm{~nm}$ of side chain trans-azobenzene chromophores in poly(MtMA-co-MAB)s in all trans configuration and at the photostationary state

\begin{tabular}{|c|c|c|c|c|c|c|c|}
\hline \multirow{3}{*}{ Sample } & \multirow{3}{*}{$\frac{\mathrm{MAB}}{\mathrm{mol} \%}$} & \multicolumn{2}{|c|}{ All trans samples ${ }^{a}$} & \multicolumn{4}{|c|}{ At the photostationary state ${ }^{a, b}$} \\
\hline & & $\lambda_{\max }$ & $\Delta \varepsilon_{\max }{ }^{c}$ & $\lambda_{\max }$ & $\Delta \varepsilon_{\max }^{\mathrm{c}}$ & $\lambda_{\max }$ & $\Delta \varepsilon_{\max }{ }^{c}$ \\
\hline & & $\mathrm{nm}$ & $\mathrm{nm}$ & $\mathrm{nm}$ & $\mathrm{nm}$ & $\mathrm{nm}$ & $\mathrm{nm}$ \\
\hline MA2 & 88.6 & 450 & -0.013 & 430 & +0.02 & - & 0 \\
\hline MA3 & 74.9 & 450 & -0.019 & 430 & +0.08 & 300 & -0.04 \\
\hline MA4 & 55.4 & 450 & -0.040 & 435 & +0.21 & 295 & -0.08 \\
\hline MA5 & 38.1 & 450 & -0.061 & 435 & +0.39 & 300 & -0.15 \\
\hline MA6 & 19.6 & 450 & -0.095 & 435 & +0.62 & 300 & -0.20 \\
\hline MA7 & 7.1 & 445 & -0.145 & 435 & +0.90 & 295 & -0.26 \\
\hline
\end{tabular}

a In chloroform solution at room temperature

b Under irradiation at $348 \mathrm{~nm}$.

c Expressed in $1 \cdot \mathrm{d} \mathrm{mol}^{-1} \cdot \mathrm{cm}^{-1}$ and referred to one MAB monomeric unit.

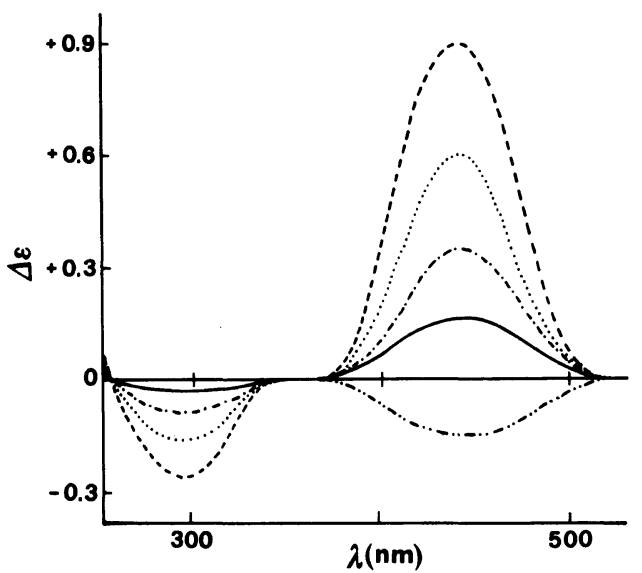

Figure 8. CD spectra at different cis/trans contents of a sample of poly(MtMA-co-MAB) containing $7.1 \%$ of MAB units: (-.- $100 \%$, (-) $91 \%,(---) 67 \%$, (----) $47 \%$, and (---) $14 \%$ of MAB units in the trans form.

isomerization seems to indicate the occurrence of a main chain conformational change of at least short chain section which should involve the modification of the whole macromolecule tertiary structure.

\section{CONCLUSION}

Copolymerization of MAB and MtMA by free radical initiation gives rise to copolymer
Table VI. Circular dichroism of the $n \rightarrow \pi^{*}$ electronic transition of side chain azobenzene chromophores in a sample of poly(MtMA-co-MAB) containing $7.1 \%$ MAB units at different content of trans-MAB units

\begin{tabular}{cc}
\hline trans-MAB units $^{\mathrm{a}}$ & $\Delta \varepsilon^{\mathrm{b}}$ \\
\cline { 1 - 1 }$\%$ & \\
\hline 100 & -0.15 \\
91 & +0.17 \\
67 & +0.35 \\
47 & +0.60 \\
27 & +0.83 \\
14 & +0.90 \\
\hline
\end{tabular}

a Evaluated by assuming that at the photostationary state half of the absorbance at $320 \mathrm{~nm}$ is attributable to residual trans-MAB units.

b Expressed in $1 \cdot \mathrm{d} \mathrm{mol}^{-1} \cdot \mathrm{cm}^{-1}$ and referred to one MAB monomeric unit.

samples having a substantially random distribution of monomeric units. Both trans to cis photoisomerization and cis to trans thermal isomerization of poly(MtMA-co-MAB)s in all cases follow the first order kinetics and are independent of both polymer chemical composition and macromolecular structure. This result can be attributed either to the relative distance from main chain and rotational freedom of the side-chain azobenzene chromo- 
phores or to an isomerization mechanism occurring through a linear transition state without involving large molecular motions. ${ }^{25}$

The CD spectra of copolymer samples in all trans configuration exhibit only a dichroic band connected with the $n \rightarrow \pi^{*}$ electronic transition, whereas at the photostationary state CD bands are present in the spectral region of both $n \rightarrow \pi^{*}$ and $\pi \rightarrow \pi^{*}$ transitions. The observed dependence of the ellipticity of the $430 \mathrm{~nm}$ band on the extent of photoisomerization could be attributed to the occurence of a photoinduced tertiary structure variation.

The monotonic dependence of ellipticity on copolymer chemical composition and comparison with the $C D$ data reported for the corresponding acrylic copolymers ${ }^{1}$ suggest that only isolated MAB units are responsible of the observed $C D$ features, the chiral perturbation induced by MtMA units being not transmitted along MAB sequences. The different $C D$ behaviour observed in acrylic and methacrylic polymers may be attributed to variations of either distribution of monomeric units or main chain tacticity. However, an influence of the methyl substituent in the main chain on the conformational arrangement of macromolecules in solution cannot be ruled - out. Synthesis of stereoregular copolymer samples in due course can better clarify this point.

Acknowledgments. The authors wish to express their appreciation to Dr. A. Fissi for the skillful assistance given in recording $C D$ spectra. The partial financial support from Ministery of Public Education $(60 \%)$ is gratefully acknowledged.

\section{REFERENCES}

1. A. Altomare, C. Carlini, F. Ciardelli, R. Solaro, and N. Rosato, J. Polym. Sci., Polym. Chem. Ed., 22, 1267 (1984).
2. A. Altomare, C. Carlini, and R. Solaro, Polymer, 23, 1355 (1982).

3. A. Altomare, C. Carlini, M. Panattoni, and R. Solaro, Macromolecules, 17, 2207 (1984).

4. A. Altomare, C. Carlini, F. Ciardelli, R. Solaro, J. L. Houben, and N. Rosato, Polymer, 24, 95 (1983).

5. A. Altomare, C. Carlini, F. Ciardelli, M. Panattoni, R. Solaro, and J. L. Houben, Macromolecules, 18, 729 (1985).

6. F. Ciardelli, C. Carlini, R. Solaro, A. Altomare, O. Pieroni, J. L. Houben, and A. Fissi, Pure \& Appl. Chem., 56, 329 (1984).

7. R. N. Majumdar and C. Carlini, Makromol. Chem., 181, 201 (1980).

8. E. Chiellini, R. Solaro, F. Ciardelli, G. Galli, and A. Ledwith, Polymer Bull., 2, 577 (1980).

9. E. Chiellini, R. Solaro, G. Galli, and A. Ledwith, Macromolecules, 13, 1654 (1980).

10. G. Galli, R. Solaro, E. Chiellini, and A. Ledwith, Macromolecules, 16, 497 (1983).

11. H. Sobue, K. Matsuzaki, and S. Nakano, J. Polym. Sci., A, 2, 3339 (1964).

12. K. Matsuzaki, A. Ishida, and N. Tateno, J. Polym. Sci., C, 16, 2111 (1967).

13. T. Kelen and F. Tudos, J. Makromol. Sci. Chem., 9, 1 (1975).

14. G. E. Ham, "High polymers: copolymerization," Vol. XVIII, G. E. Ham, Ed., Interscience, New York, N. Y., 1964, p 4.

15. F. R. Mayo and C. Walling, Chem. Rev., 200 (1950).

16. E. Chiellini, R. Solaro, O. Colella, and A. Ledwith, Eur. Polym. J., 14, 489 (1978).

17. R. Solaro, G. Galli, A. Ledwith, and E. Chiellini, "Polymer Photophysics," D. Phillips, Ed., Chapman and Hall, London, 1985, p 377.

18. J. L. Houben, A. Fissi, D. Bacciola, N. Rosato, O. Pieroni, and F. Ciardelli, Int. J. Biol. Macromol., 5, 94 (1983).

19. H. H. Jaffe and M. Orchin, "Theory and Application of Ultraviolet Spectroscopy," Wiley, New York, N. Y., 1962.

20. C. Carlini and F. Gurzoni, Polymer, 24, 101 (1983).

21. I. Tinoco, Jr., J. Am. Chem. Soc., 82, 4785 (1960).

22. K. Okamoto, A. Itaya, and S. Kusabayashi, Chem. Lett., 1167 (1974).

23. R. N. Majumdar, C. Carlini, N. Rosato, and J. L. Houben, Polymer, 21, 941 (1980).

24. R. N. Majumdar, C. Carlini, and C. Bertucci, Makromol. Chem., 183, 2047 (1982).

25. D. L. Ross and J. Blanc, "Photochromism," C. H. Brown Ed., Wiley, New York, N. Y., p 471. 